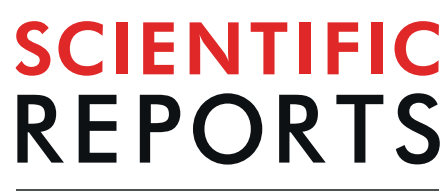

natureresearch

\title{
Resveratrol and its Related Polyphenols Contribute to the Maintenance of Genome Stability
}

Yusuke Matsuno ${ }^{1,2}$, Yuko Atsumi ${ }^{1}$, Md. Alauddin ${ }^{3}$, Md. Masud Rana ${ }^{3}$, Haruka Fujimori ${ }^{1,4}$, Mai Hyodo ${ }^{1,4}$, Atsuhiro Shimizu ${ }^{1}$, Tomoki Ikuta ${ }^{5}$, Hiroko Tani ${ }^{5}$, Hidetaka Torigoe ${ }^{1,2}$, Yoshimichi Nakatsu ${ }^{6}$, Teruhisa Tsuzuki ${ }^{6}$, Michio Komai $^{3}$, Hitoshi Shirakawa ${ }^{3,7}$ \& Ken-ichiYoshioka ${ }^{1 *}$

Genomic destabilisation is associated with the induction of mutations, including those in cancer-driver genes, and subsequent clonal evolution of cells with abrogated defence systems. Such mutations are not induced when genome stability is maintained; however, the mechanisms involved in genome stability maintenance remain elusive. Here, resveratrol (and related polyphenols) is shown to enhance genome stability in mouse embryonic fibroblasts, ultimately protecting the cells against the induction of mutations in the ARF/p53 pathway. Replication stress-associated DNA double-strand breaks (DSBs) that accumulated with genomic destabilisation were effectively reduced by resveratrol treatment. In addition, resveratrol transiently stabilised the expression of histone $\mathrm{H} 2 \mathrm{AX}$, which is involved in DSB repair. Similar effects on the maintenance of genome stability were observed for related polyphenols. Accordingly, we propose that polyphenol consumption can contribute to the suppression of cancers that develop with genomic instability, as well as lifespan extension.

Most cancers are associated with genomic instability, which can be categorised as chromosomal instability or microsatellite instability (MSI) ${ }^{1}$. Genomic destabilisation is a major cause of mutations, including those in cancer-driver genes, and can lead to clonal evolution of cells with abrogated defence systems, such as those containing mutations in the ARF/p53 pathway ${ }^{2}$. Genome stability maintenance would likely prevent the formation of mutations and suppress cancer development; however, it is still unclear if genome stability can be maintained in vivo and whether this process can indeed suppress the occurrence of cancer.

Genomic instability is caused by the erroneous repair of DNA double-strand breaks (DSBs); paradoxically, the DNA repair systems of most cancers that develop with genomic instability are genetically normal ${ }^{3}$. The mechanisms by which normal cells accumulate DSBs remain unclear, but DSBs widely accumulate in pre-cancerous cells and are accompanied by genomic instability ${ }^{2,4-6}$. In vitro, replication stress-associated DSBs and the associated genomic instability are observed in cells subjected to aberrant growth stimulation ${ }^{2}$ or overexpression of oncogenes such as c-Myc and $\mathrm{E} 2 \mathrm{~F} 1^{6-8}$.

Possibly reflecting the correlation between cancer development and age, DSBs accumulate with age in vivo and with cultivating passages in vitro ${ }^{9}$, suggesting that ageing cells are defective in DSB repair. DSB repair deficiency is probably related at least in part to a reduction in the level of H2AX. This histone mediates DSB repair and is required for genome stability maintenance, and $\mathrm{H} 2 \mathrm{AX}$ expression levels are attenuated when the growth rate of normal cells slows down ${ }^{10,11}$. In fact, such cells are defective in repairing replication stress-associated DSBs ${ }^{10}$,

${ }^{1}$ Division of Carcinogenesis and Cancer Prevention, National Cancer Center Research Institute, 5-1-1 Tsukiji, Chuo-ku, Tokyo, 104-0045, Japan. ${ }^{2}$ Department of Applied Chemistry, Faculty of Science, Tokyo University of Science, 1-3 Kagurazaka, Shinjuku-ku, Tokyo, 162-8601, Japan. ${ }^{3}$ Laboratory of Nutrition, Graduate School of Agricultural Science,

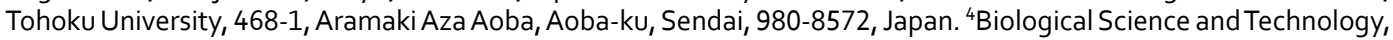
Tokyo University of Science, 6-1-1 Niijuku, Katsushika-ku, Tokyo, 125-8585, Japan. Institute for Bee Products and Health Science, Yamada Bee Company, Inc., 194 Ichiba, Kagamino-cho, Tomata-gun, Okayama, 708-0393, Japan. ${ }^{6}$ Department of Medical Biophysics and Radiation Biology, Faculty of Medical Sciences, Kyushu University, Maidashi, Higashi-ku, Fukuoka, 812-8582, Japan. ${ }^{7}$ International Education and Research Center for Food and Agricultural Immunology, Graduate School of Agricultural Science, Tohoku University, 468-1 Aramaki Aza Aoba, Aoba-ku, Sendai, 980-8572, Japan. *email: kyoshiok@ncc.go.jp 

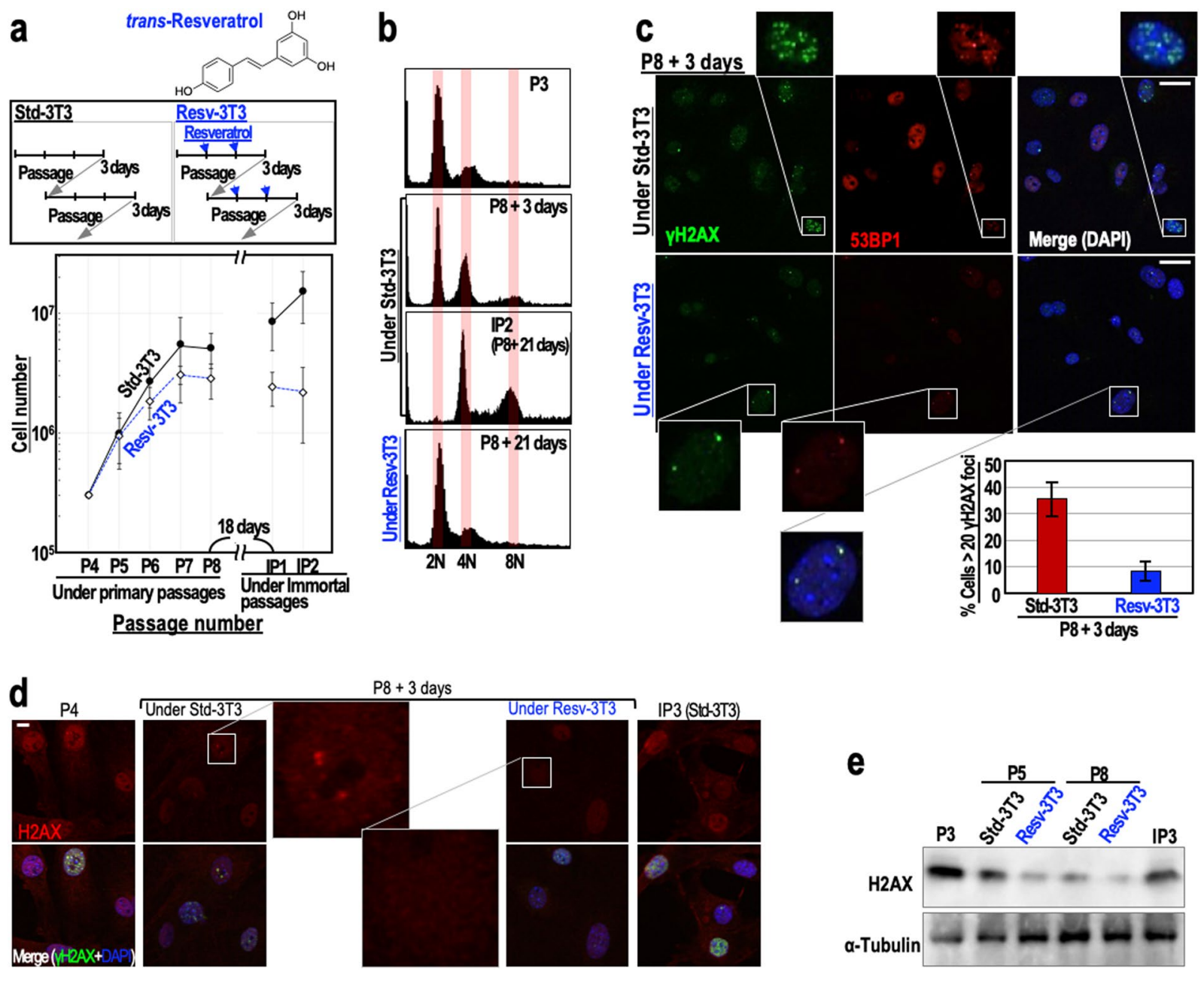

Figure 1. Regular resveratrol treatment maintains genome stability and suppresses DSB accumulation. (a) The immortalisation of MEFs cultivated under the Std-3T3 or Resv-3T3 protocol as indicated. In the Resv-3T3 protocol, the cells were treated with $2.5 \mu \mathrm{M}$ resveratrol regularly. In the lower graph, data are represented as the mean \pm s.d. ( $n=3$ biologically independent experiments). (b) The effects of cultivation of MEFs under the Std-3T3 or Resv-3T3 protocol on chromosomal instability (tetraploidy). (c) Statuses of the $\gamma \mathrm{H} 2 \mathrm{AX}$ and 53BP1 foci in MEFs at a growth-arrested stage (P8 + 3 days) following cultivation under the Std-3T3 or Resv-3T3 protocol. The numbers of $\gamma \mathrm{H} 2 \mathrm{AX}$ foci were quantified and data are represented as the mean $\pm \mathrm{s}$.d. $(\mathrm{n}=3$ biologically independent experiments). (d,e) Immunostaining (d) and immunoblot (e) analyses of H2AX in quiescent MEFs grown under the Std-3T3 or Resv-3T3 protocol. (c,d) Scale bars, $10 \mu \mathrm{m}$.

although they are still able to repair DSBs caused by $\gamma$-rays because H2AX is transiently stabilised under these conditions ${ }^{12}$.

A number of animal studies have shown that regular polyphenol consumption can contribute to cancer suppression in association with lifespan extension ${ }^{13-18}$. Such cancer-suppressive effects of polyphenols have been reported for a wide variety of cancers that generally arise with genomic instability at advanced ages, such as skin ${ }^{19}$, prostate $^{20}$, colon $^{21}$ and breast cancers ${ }^{22}$. It is possible that the anti-cancer and anti-aging effects of polyphenols are related to their positive effects on genome integrity.

In this study, we examined the effects of polyphenols on genome stability maintenance, DSB repair and genomic instability-associated cancer suppression. We found that resveratrol, a polyphenol found in red wine, and related polyphenols maintain genome stability by inducing DSB repair, thereby contributing to the suppression of cancer that develops with genomic instability.

\section{Results}

Resveratrol contributes to genome stability. To examine the effects of resveratrol on genome stability, we monitored the immortalisation of mouse embryonic fibroblasts (MEFs) (Fig. 1a). When cultivated under the '3T3 protocol', MEFs initially show serial proliferation, but then undergo growth-arrested senescence, and subsequently immortalise with genomic instability (tetraploidy) ${ }^{8,10}$ and mutations in the ARF/p53 pathway ${ }^{11,23}$. As expected, MEFs grown under the standard 3T3 protocol (Std-3T3) immortalised with tetraploidy, but MEFs that were regularly treated with $2.5 \mu \mathrm{M}$ resveratrol (Resv-3T3 protocol) displayed continuous genome stability 
and were protected against immortalisation (Fig. 1a,b). Supporting these results, the percentages of cells with two nuclei or micronuclei, the levels of which increase during genomic destabilisation, were lower for the Resv-3T3 group than for the Std-3T3 group (Supplementary Fig. S1a). The induction of aberrant nuclei by $\gamma$-ray irradiation was also reduced in the presence of resveratrol, although the differences between the control cells and resveratrol-treated cells were not statistically significant (Supplementary Fig. S1b). These results indicate that continuous resveratrol treatment contributes to genome stability maintenance. Given that MEFs generally immortalise with abrogation of the ARF/p53-dependent barrier ${ }^{11,23}$, resveratrol may suppress the induction of mutations in cancer-driver genes via the maintenance of genome stability.

Genomic destabilisation (tetraploidisation) in MEFs is generally triggered by replication stress-associated DSBs that accumulate under the Std-3T3 protocol, in association with cellular stress caused by continuous growth acceleration. Since our initial experiment suggested that regular resveratrol treatment can suppress genomic destabilisation, we examined the accumulation of DSBs in passage 8 (P8) MEFs grown under the Std-3T3 and Resv-3T3 protocols, by counting the numbers of $\gamma \mathrm{H} 2 \mathrm{AX} / 53 \mathrm{BP} 1$ foci. As expected, the number of $\gamma \mathrm{H} 2 \mathrm{AX} / 53 \mathrm{BP} 1$ foci in MEFs grown under the Resv-3T3 protocol was markedly smaller than that in MEFs grown under the Std-3T3 protocol (Fig. 1c). This finding is consistent with the observed genome stabilising effect of resveratrol described above, and suggests that regular resveratrol treatment is associated with the reduction of DSBs and the neutralisation of replication stress.

Normal cells generally undergo growth arrest after serial proliferation. At this stage, two different cellular states can be discriminated: (1) a senescent state associated with $\gamma \mathrm{H} 2 \mathrm{AX}$ foci accumulation and an increased risk of genomic destabilisation, and (2) a quiescent state associated with down-regulated H2AX expression and maintenance of genome stability ${ }^{24}$. Therefore, we compared the expression levels of H2AX in MEFs grown under the Std-3T3 and Resv-3T3 protocols. As expected, an immunostaining analysis revealed that the H2AX signal was substantially lower in the Resv-3T3 cells than in the Std-3T3 cells at the growth-arrested stage (P8 + 3 days) (Fig. 1d). A western blotting analysis confirmed that, as seen in quiescent cells, H2AX expression was down-regulated in both cell groups at the growth-arrested stage, but the reduction was larger for the resveratrol-treated cells than for those grown under the standard protocol (Fig. 1e). Together with the observed reduction in $\gamma \mathrm{H} 2 \mathrm{AX} / 53 \mathrm{BP} 1$ foci formation following resveratrol treatment, these findings indicate that resveratrol contributes to DSB reduction and genome stability maintenance by inducing a quiescent state with down-regulated $\mathrm{H} 2 \mathrm{AX}$ levels.

Resveratrol enables DSB repair through transient induction of $\mathrm{H} 2 \mathrm{AX}$. After P8, MEFs are at an increased risk of genomic destabilisation due to a deficiency in the repair of DSBs caused by the replication stress that arises during cultivation under Std-3T3 conditions. Although it is unclear how normal cells become defective in DSB repair after serial proliferation, the process is thought to involve a reduction in $\mathrm{H} 2 \mathrm{AX}$ levels ${ }^{10,11}$. It is possible that the repair of DSBs in such cells occurs via transient up-regulation of H2AX, as occurs after $\gamma$-ray irradiation of cells $\mathrm{s}^{12}$. To test this hypothesis, we performed a $24 \mathrm{hr}$ time-course analysis of H2AX levels in MEFs treated with $5 \mu \mathrm{M}$ resveratrol (Fig. 2a,b). As expected, $\mathrm{H} 2 \mathrm{AX}$ was transiently induced after resveratrol treatment of $\mathrm{P} 8 \mathrm{MEFs}$, with the peak induction at $6 \mathrm{hr}$ post-treatment. In addition, $\mathrm{H} 2 \mathrm{AX}$ transiently induced by resveratrol was efficiently incorporated into chromatin but its level decayed within $24 \mathrm{hr}$ (Fig. 2c).

To examine its effect on DSB repair, we treated P8 MEFs with different concentrations of resveratrol and monitored $\gamma \mathrm{H} 2 \mathrm{AX}$ foci status. As expected, the number of $\gamma \mathrm{H} 2 \mathrm{AX}$ foci was reduced significantly $24 \mathrm{hr}$ after treatment of MEFs with $2.5 \mu \mathrm{M}$ resveratrol (Fig. 2d). In addition, the number of $\gamma \mathrm{H} 2 \mathrm{AX}$ foci was reduced further by multiple resveratrol treatments (Fig. 2e). The $\gamma \mathrm{H} 2 \mathrm{AX}$ foci largely merged with 53BP1 foci, which accumulate at DSB sites (Fig. 2f), and with phosphorylated RPA32 (p-RPA at Ser33) foci, which arise in association with replication stress (Fig. 2g). The numbers of these foci were reduced in the presence of resveratrol (Fig. 2f), suggesting efficient repair of DSBs in the presence of resveratrol.

Resveratrol may modulate multiple biological pathways and may induce apoptosis at high doses ${ }^{25}$. To compare the conditions that lead to DSB repair and apoptosis, the effects of three concentrations of resveratrol (2.5, 25 and $250 \mu \mathrm{M}$ ) on multiple cell types were examined after pre-treatment of cells with $0.25 \mathrm{mM}$ hydroxyurea to weakly induce replication stress. As expected, the level of cleaved caspase-3, a marker of apoptosis induction, was increased after treatment of MEFs (P4), HUC-F2 cells (P9) and WI38 cells (P6) with $250 \mu \mathrm{M}$ resveratrol, and after treatment of HeLa cells with $25 \mu \mathrm{M}$ resveratrol (Supplementary Fig. S2a). Under these conditions, the numbers of $\gamma \mathrm{H} 2 \mathrm{AX}$ foci were not increased prior to the induction of apoptosis (Supplementary Fig. S2b; see $25 \mu \mathrm{M}$ for $\mathrm{HeLa}$ and $250 \mu \mathrm{M}$ for MEFs), implying the separation of apoptosis induction from damage responses. Notably, these findings indicate that the concentration of resveratrol that leads to DSB reduction is much lower than that required for the induction of apoptosis.

Normal cells treated with hydroxyurea usually undergo growth arrest and hence show only a limited response to damage ${ }^{26}$. Therefore, we also examined the effect of resveratrol on the numbers of replication stress-associated DSBs $24 \mathrm{hr}$ after $\gamma$-ray irradiation (2 Gy) of MEFs, HeLa cells and WI38 cells (Supplementary Fig. S3a), because replication stress-associated DSBs accumulate after the repair of DSBs that are directly caused by $\gamma$-ray exposure $^{27}$. As expected, the number of $\gamma \mathrm{H} 2 \mathrm{AX}$ foci that merged with p-RPA (Supplementary Fig. 3b) and 53BP1 was reduced significantly in the presence of $2.5 \mu \mathrm{M}$ resveratrol (Supplementary Fig. S3a). Furthermore, the numbers of $\gamma \mathrm{H} 2 \mathrm{AX}$ foci and 53BP1 foci in MEFs that were $\gamma$-ray irradiated with 1 or 5 Gy were also reduced significantly in the presence of $2.5 \mu \mathrm{M}$ resveratrol (Supplementary Fig. S3c). Based on these findings, we concluded that replication stress-associated DSBs are reduced by treatment of cells with relatively low concentrations of resveratrol.

Resveratrol-related polyphenols contribute to genome stability. A number of studies have reported potential benefits of polyphenol consumption on cancer suppression and longevity ${ }^{28-30}$. Therefore, we examined the genome stabilising effects of chlorogenic acid, a polyphenol found in coffee, and melinjo resveratrol, 
a Resveratrol treatment for $1 \mathrm{hr}$

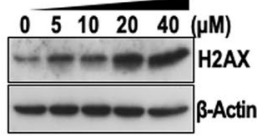

b 5 uM Resveratrol treatment $\begin{array}{lllllll}0 & 1.5 & 3 & 6 & 12 & 24 & (\mathrm{hr})\end{array}$

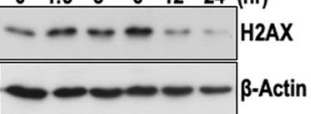
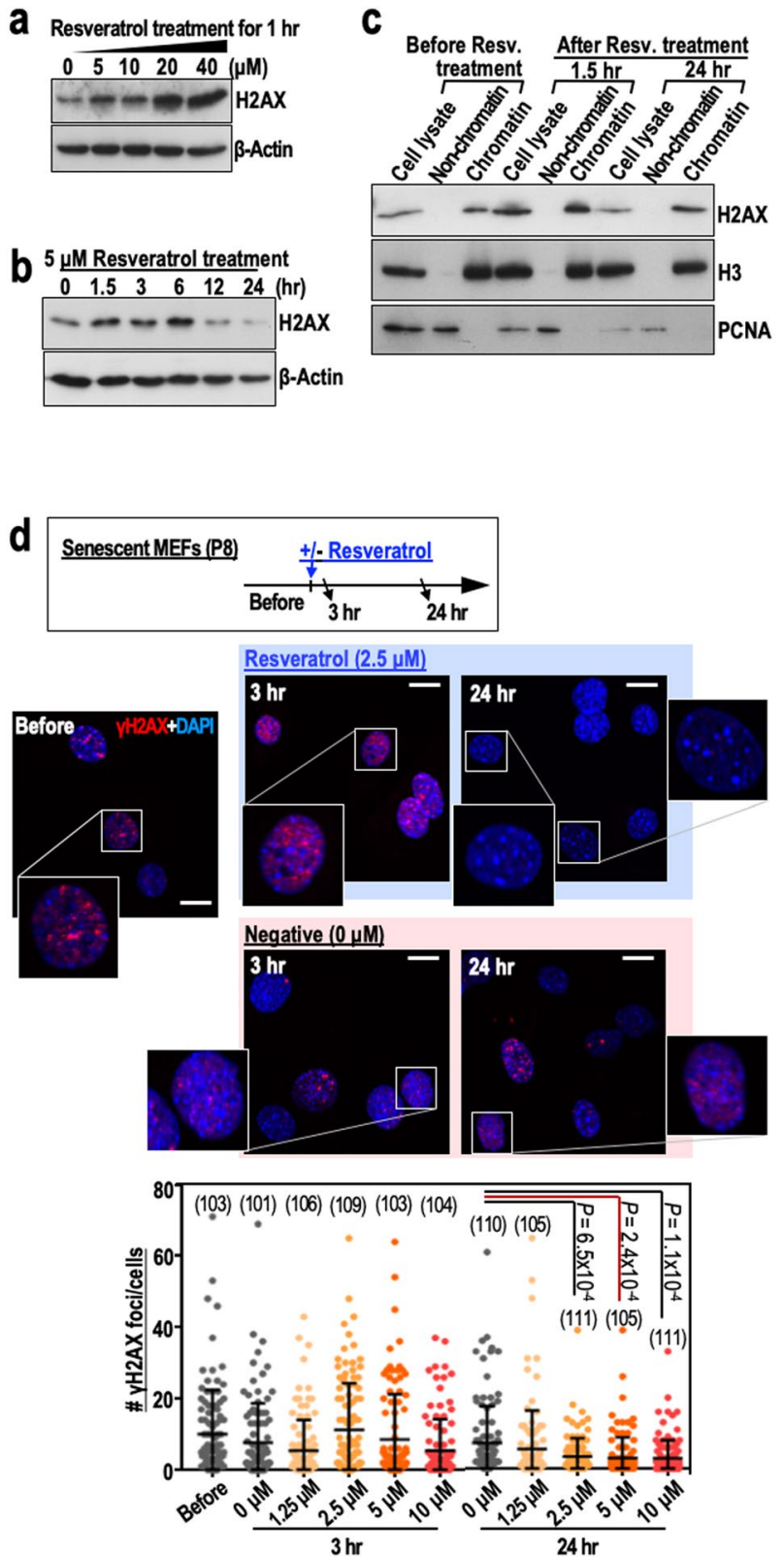

e
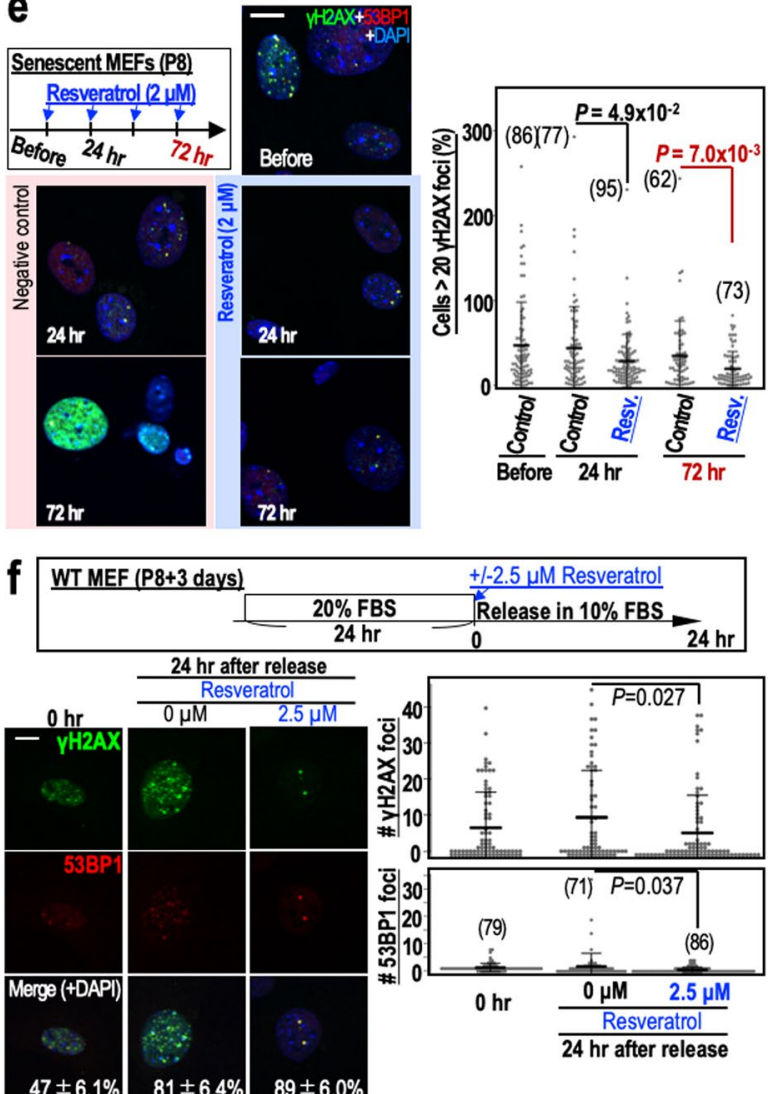

g
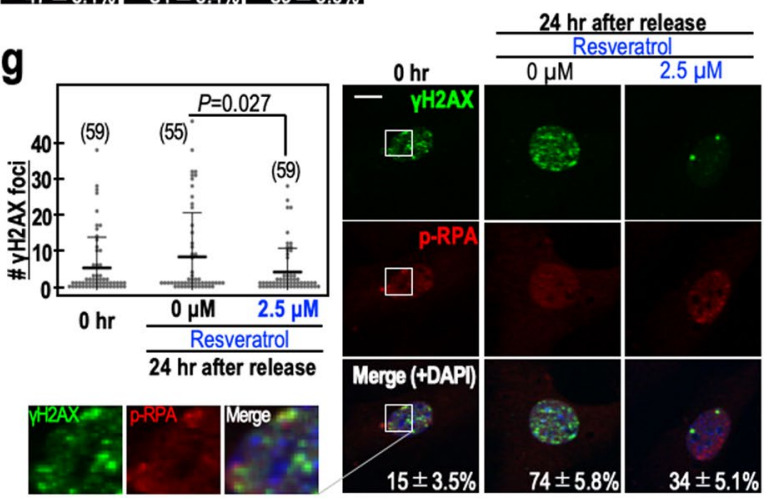

Figure 2. Resveratrol treatment of MEFs transiently stabilises $\mathrm{H} 2 \mathrm{AX}$, leading to reduced numbers of $\gamma \mathrm{H} 2 \mathrm{AX}$ foci. (a) H2AX expression in senescent MEFs (passage 8) treated with different concentrations of resveratrol. The dose-dependent effects of resveratrol were assessed $1 \mathrm{hr}$ after treatment. (b) The time-dependent effects of $5 \mu \mathrm{M}$ resveratrol on $\mathrm{H} 2 \mathrm{AX}$ expression in senescent MEFs (passage 8). (c) H2AX expression in senescent MEFs (passage 8) treated with resveratrol $(5 \mu \mathrm{M})$ and then fractionated into the chromatin and non-chromatin fractions before and 1.5 and $24 \mathrm{hr}$ after treatment. Histone $\mathrm{H} 3(\mathrm{H} 3)$ and PCNA were used as controls.

(d) Immunofluorescence analyses examining the effect of resveratrol $(2.5 \mu \mathrm{M})$ on the number of $\gamma \mathrm{H} 2 \mathrm{AX}$ foci in senescent MEFs ( $\mathrm{n}$ numbers are indicated in the graph). The dose-dependent effects of resveratrol were assessed 3 and $24 \mathrm{hr}$ after treatment. (e) The effects of multiple resveratrol treatments $(2 \mu \mathrm{M})$ on the number of $\gamma \mathrm{H} 2 \mathrm{AX}$ foci in senescent MEFs. Reductions in the number of $\gamma \mathrm{H} 2 \mathrm{AX}$ foci were assessed $24 \mathrm{hr}$ (single treatment) and $72 \mathrm{hr}$ (three treatments) after resveratrol exposure. Data are represented as the mean \pm s.d. $(\mathrm{n}=3$ biologically independent experiments). (f,g) The effects of resveratrol $(2.5 \mu \mathrm{M})$ on the numbers of merged $\gamma \mathrm{H} 2 \mathrm{AX} / 53 \mathrm{BP} 1$ (f) and $\gamma \mathrm{H} 2 \mathrm{AX} / \mathrm{p}$-RPA (g) foci in senescent MEFs. The MEFs were treated as shown in the workflow (f). $\gamma \mathrm{H} 2 \mathrm{AX}, 53 \mathrm{BP} 1$ and $\mathrm{p}-\mathrm{RPA}$ were detected by immunofluorescence (n numbers are indicated in the graph). The percentages of the $\gamma \mathrm{H} 2 \mathrm{AX}$ foci that merged with 53BP1 or p-RPA foci (mean \pm s.e.) are indicated in each image. Data in the graphs are represented as the mean \pm s.d. Scale bars, $10 \mu \mathrm{m}$. $P$-values were calculated by two-tailed Welch's $t$-tests.

a mixture of multiple resveratrol-associated polyphenols produced in melinjo seeds. Similar to resveratrol (Fig. 1), chlorogenic acid $(2.5 \mu \mathrm{M})$ and melinjo resveratrol $(0.5 \mu \mathrm{g} / \mathrm{ml})$ inhibited the immortalisation of MEFs (Fig. 3a) and promoted genome stability (Fig. 3b). In addition, as seen for resveratrol, chlorogenic acid and melinjo resveratrol 

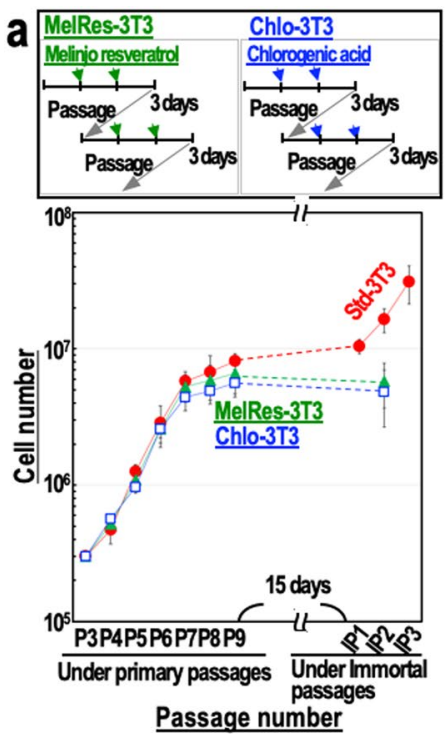

Passage number

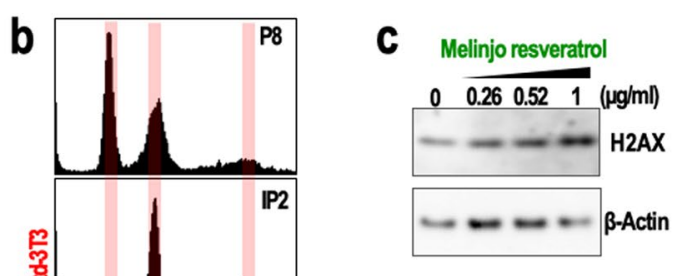

Melinjo resveratrol $(0.5 \mu \mathrm{g} / \mathrm{ml})$

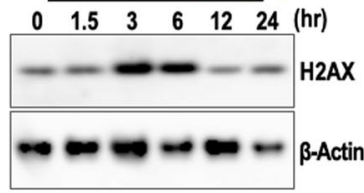

d
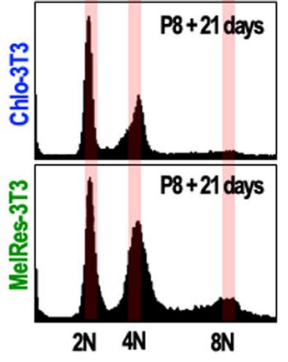

$5 \mu \mathrm{M}$ Chlorogenic acid

\begin{tabular}{llllll}
\hline 0 & 1.5 & 3 & 6 & 12 & 24 \\
\hline
\end{tabular}

$\begin{array}{lllll}0 & 1.25 & 2.5 & 5 & 10(\mu \mathrm{M})\end{array}$

$-=-\cdots$ H2AX

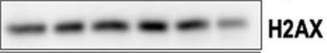

$\sim-\infty$ B-Actin H2AX $\beta$-Actin
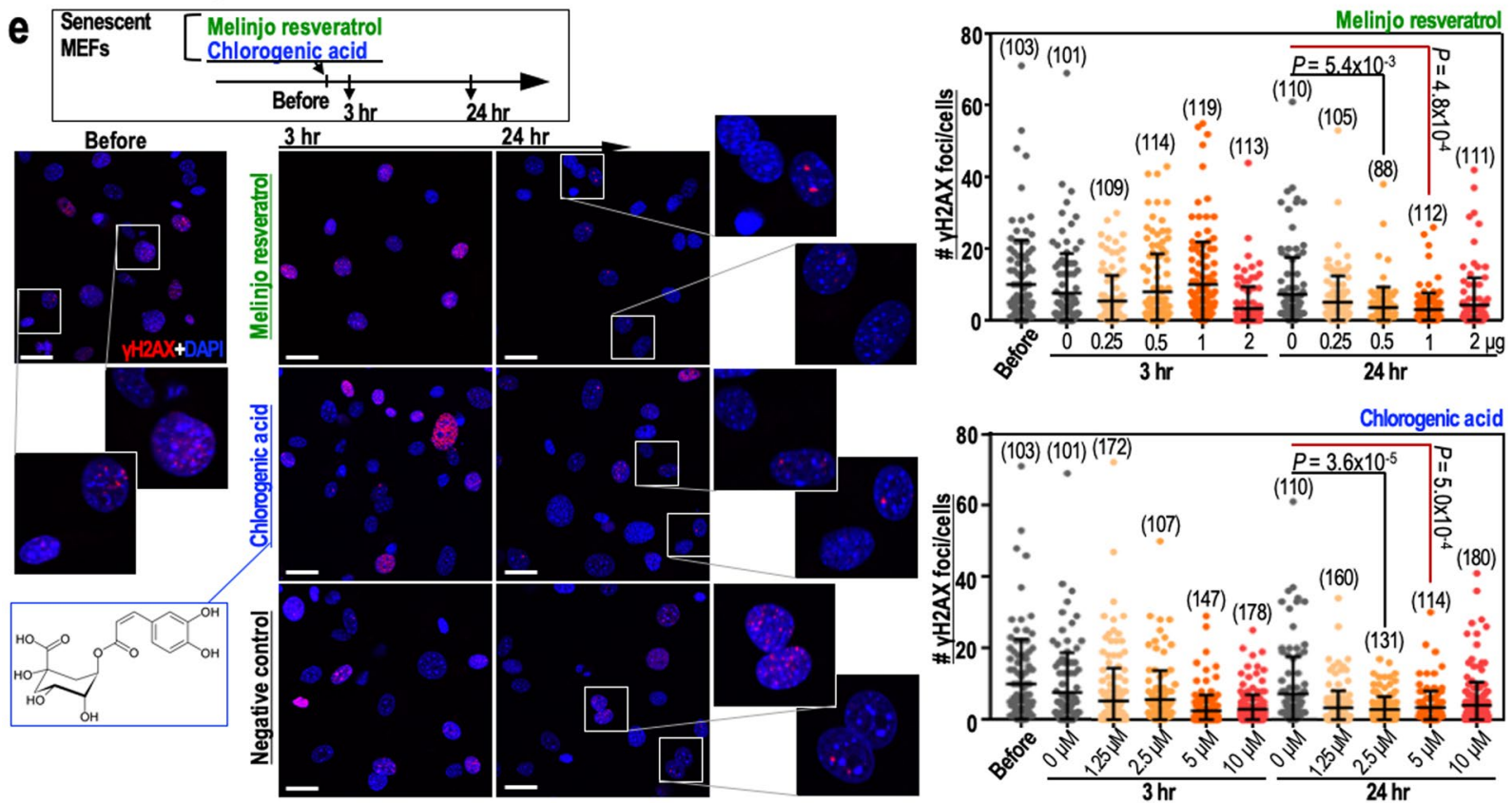

Figure 3. Melinjo resveratrol and chlorogenic acid contribute to genome stability maintenance. (a) The immortalisation of MEFs cultivated under the Std-3T3, MelRes-3T3 (melinjo resveratrol) or Chlo-3T3 (chlorogenic acid) protocol as indicated. In the MelRes-3T3 and Chlo-3T3 protocols, MEFs were regularly treated with $0.5 \mu \mathrm{g} / \mathrm{ml}$ melinjo resveratrol and $2.5 \mu \mathrm{M}$ chlorogenic acid, respectively. In the lower graph, data are represented as the mean \pm s.d. ( $n=3$ biologically independent experiments). (b) The effects of cultivation of MEFs under the Std-3T3, MelRes-3T3 or Chlo-3T3 protocol on chromosomal instability (tetraploidy). (c,d) Time- and dose-dependent effects of melinjo resveratrol (c) and chlorogenic acid (d) on H2AX expression in senescent MEFs (passage 8). The dose-dependent effects were assessed $3 \mathrm{hr}$ after treatment. The time-dependent effects were determined using $0.5 \mu \mathrm{g} / \mathrm{ml}$ melinjo resveratrol (c) or $5 \mu \mathrm{M}$ chlorogenic acid (d). (e) The effects of melinjo resveratrol $(0.5 \mu \mathrm{g} / \mathrm{ml})$ and chlorogenic acid $(5 \mu \mathrm{M})$ on the number of $\gamma \mathrm{H} 2 \mathrm{AX}$ foci in senescent MEFs at 3 and $24 \mathrm{hr}$ post-treatment. $\gamma \mathrm{H} 2 \mathrm{AX}$ foci were detected by immunofluorescence (n numbers are indicated in the graphs). Data in the graphs are represented as the mean $\pm \mathrm{s}$.d. Scale bars, $10 \mu \mathrm{m}$. $P$-values were calculated by two-tailed Welch's $t$-tests.

caused a transient induction of $\mathrm{H} 2 \mathrm{AX}$ (Fig. 3c,d) and a reduction in the number of $\gamma \mathrm{H} 2 \mathrm{AX}$ foci (Fig. 3e). These findings suggest that the broad health benefits of these polyphenols could be due to the maintenance of genome stability, which primarily occurs via the reduction of DSBs.

Melinjo resveratrol is a mixture of multiple polyphenols, the major components being gnetin $\mathrm{C}$ (resveratrol dimer) and its glycosides gnemonoside $\mathrm{A}$ and gnemonoside $\mathrm{D}^{31}$. To determine which of these components is responsible for the effect of melinjo resveratrol on genome stability, we examined their effects on transient $\mathrm{H} 2 \mathrm{AX}$ induction and resulting DSB repair $(\gamma \mathrm{H} 2 \mathrm{AX}$ foci reduction) in MEFs. Gnetin C induced transient $\mathrm{H} 2 \mathrm{AX}$ 

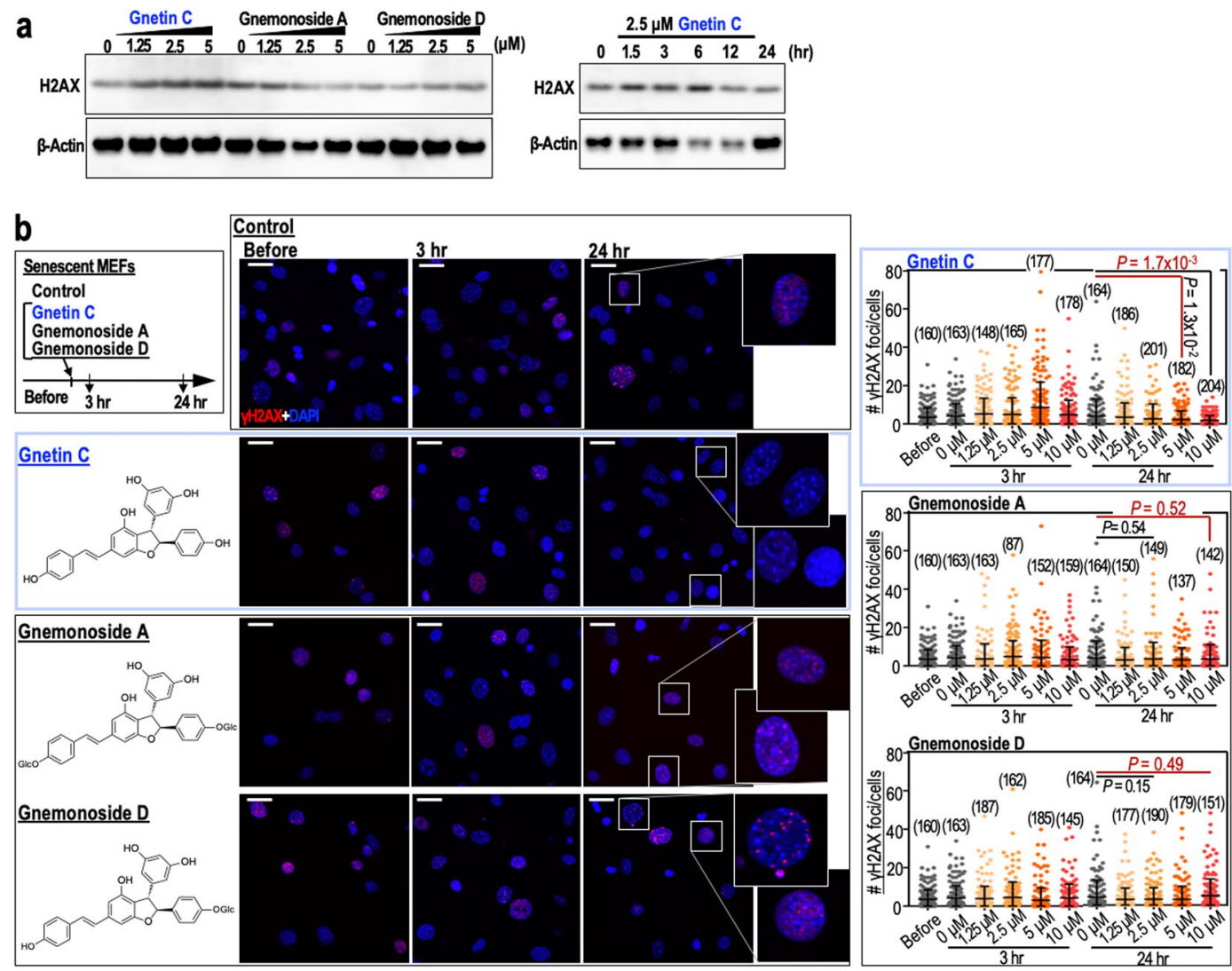

Figure 4. Gnetin $\mathrm{C}$ mediates transient stabilisation of $\mathrm{H} 2 \mathrm{AX}$ and a reduction in the number of $\gamma \mathrm{H} 2 \mathrm{AX}$ foci. (a) Dose-dependent effects of gnetin C, gnemonoside A and gnemonoside D, and time-dependent effects of gnetin $\mathrm{C}$ on $\mathrm{H} 2 \mathrm{AX}$ expression in senescent MEFs (passage 8). The dose-dependent effects were assessed $3 \mathrm{hr}$ after treatment. The time-dependent effect was determined using $2.5 \mu \mathrm{M}$ gnetin $\mathrm{C}$. (b) The effects of gnetin $\mathrm{C}$ $(2.5 \mu \mathrm{M})$, gnemonoside $\mathrm{A}(2.5 \mu \mathrm{M})$ and gnemonoside $\mathrm{D}(2.5 \mu \mathrm{M})$ on the numbers of $\gamma \mathrm{H} 2 \mathrm{AX}$ foci in senescent MEFs at 3 and $24 \mathrm{hr}$ post-treatment. Data in the graphs are represented as the mean \pm s.d. Scale bars, $10 \mu \mathrm{m} . P$ values were calculated by two-tailed Welch's $t$-tests.

induction and DSB repair in P8 MEFs, whereas gnemonoside A and gnemonoside D did not (Fig. 4a,b), indicating that the gnetin $\mathrm{C}$ component of melinjo resveratrol contributes to the reduction of DSBs in association with transient $\mathrm{H} 2 \mathrm{AX}$ induction.

Consumption of melinjo resveratrol suppresses cancer development. Based on our results demonstrating the genome stabilising effect of melinjo resveratrol, we used $M s h 2^{-/-}$mice to examine its effect on cancer suppression and its possible association with lifespan extension. $M s h 2^{-1-}$ mice have high mutation rates ${ }^{32,33}$ that predispose them to cancer (mainly lymphoma) in association with $\mathrm{MSI}^{34}$, which is related to the clonal evolution of cells containing mutations in cancer-driver genes ${ }^{2}$. Since genome stability effects of melinjo resveratrol were shown with almost same range with those of resveratrol in weight concentration (Figs. $2 \mathrm{~d}$ and $3 \mathrm{e}$ ), melinjo resveratrol were treated with same dose range with previous resveratrol studies shown cancer suppression effects ${ }^{35,36}$. Kaplan-Meir survival curves revealed that $M s h 2^{-/-}$mice fed a diet containing melinjo resveratrol had a significantly longer lifespan than those fed the control diet (Fig. 5a), indicating that melinjo resveratrol consumption contributes to lifespan extension even in a mismatch repair (MMR)-deficient background.

To evaluate the effect of melinjo resveratrol on cancer incidence, we analysed the sensitivity of $M s h 2^{-/-}$mice to $\mathrm{K}_{2} \mathrm{BrPO}_{3}$, which primarily induces DNA damage and promotes the formation of MSI-associated cancers in the small intestine ${ }^{37}$. As expected, tumour numbers were significantly lower in mice consuming the $0.03 \%$ melinjo resveratrol diet than in those consuming the control diet (Fig. 5b), in which tumours developed with MSI (Fig. 5c). These results support the notion that genomic destabilisation-associated cancers can be suppressed by the consumption of polyphenols that reduce DSBs and maintain genome stability.

\section{Discussion}

The results presented here demonstrate that resveratrol and related polyphenols contribute to genome stability maintenance, primarily via suppression of the DSB level. In an in vivo study, the beneficial effects of resveratrol on genome integrity were also associated with the suppression of MSI-associated cancer and a subsequent extension of the lifespan of mice. These results are consistent with those of previous studies reporting suppression of cancers that develop with genomic instability by polyphenol consumption ${ }^{19-22}$. 

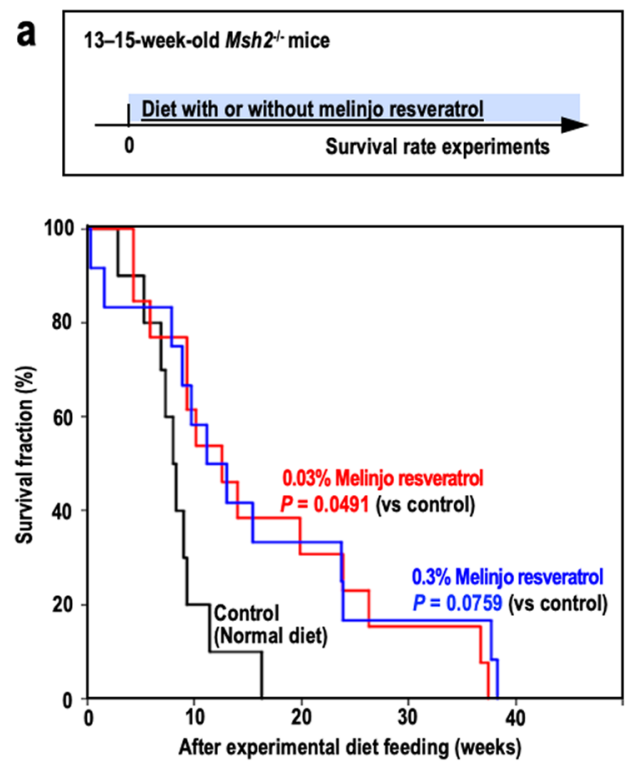
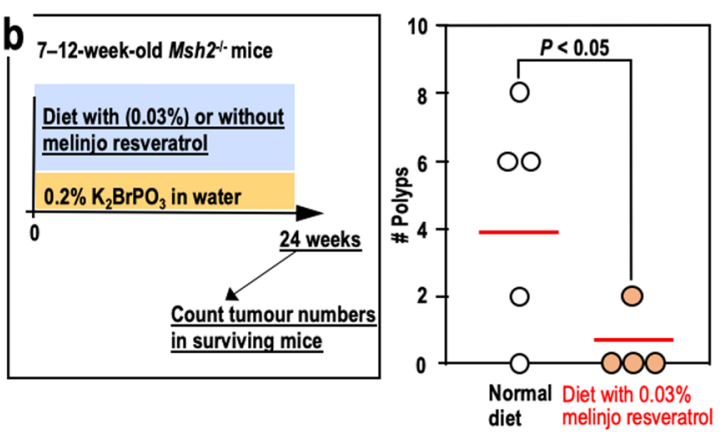

C

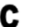

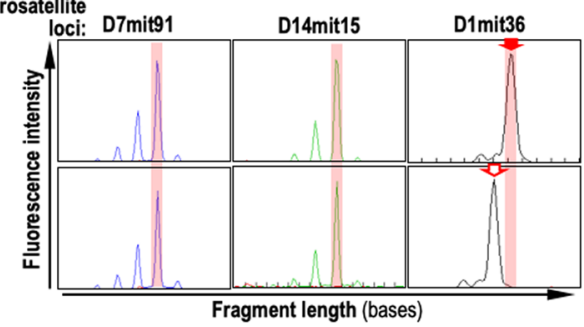

Figure 5. Melinjo resveratrol consumption contributes to the suppression of cancer associated with microsatellite instability. (a) Kaplan-Meier curves showing the survival rates of $M s h 2^{-1-}$ mice fed a normal diet or a diet supplemented with melinjo resveratrol $(0.3 \%$ or $0.03 \%)$. Data were analysed by a Log-Rank test followed by a Holm-Sidak test; $\mathrm{n}=10$ (control group), $\mathrm{n}=13(0.03 \%$ melinjo resveratrol group), $\mathrm{n}=12(0.3 \%$ melinjo resveratrol group). (b) The numbers of tumours in the small intestines of $\mathrm{K}_{2} \mathrm{BrPO}_{3}$-treated mice fed a normal diet or a diet supplemented with $0.03 \%$ melinjo resveratrol. Statistical analysis was performed via a Student's $t$-test. (c) The MSI statuses at three microsatellite loci in tissue from normal organs (top row) and polyps (bottom row). Red arrows indicate the shifted fragment peaks, i.e., MSI.

Cancer generally develops with mutations in cancer-driver genes. Whether the formation of cancer-driver mutations and the resulting disease is avoidable is still a matter of debate ${ }^{38-40}$. The conventional view is that most mutations, including cancer-driver mutations, are randomly induced during DNA replication; therefore, the majority of cancers are unavoidable ${ }^{41}$. However, a recent study reported that, unlike mutations induced during canonical replication, those caused by replication stress along with genomic destabilisation can be avoided through genome stability maintenance ${ }^{42}$. Mutations induced during canonical replication are limited, even in MMR-deficient cells that cannot repair errors caused by replication forks; however, massive numbers of mutations occur under replication stress in association with genomic destabilisation, leading to clonal evolution of cells with abrogated defence systems ${ }^{2}$. The results presented here are consistent with these observations and demonstrate that polyphenols can mediate genome stability maintenance and cancer suppression.

Because genomic instability is inevitably induced in most cancers, our results imply that many cancers are theoretically preventable through genome stability maintenance, and that polyphenol consumption is a promising preventative option. In this study, the cancer-suppressive effects of polyphenols were evident in MMR-deficient mice. Although the positive effect of polyphenols on longevity observed in this study was significant, it was still limited. To suppress cancer development and efficiently extend the healthy lifespan, it will be important to clarify the mechanisms via which genome stability is maintained.

Resveratrol directly and indirectly activates a number of factors, including SIRT1, AMPK and ATM, with multiple consequences, including positive effects on metabolism ${ }^{18}$. In addition, the results presented here demonstrate that reduction of the DSB level and maintenance of genome stability, associated with the transient stabilisation of $\mathrm{H} 2 \mathrm{AX}$, are additional effects of resveratrol. Although the mechanism by which the DSB level is reduced by resveratrol is still unclear, it appears to differ from the general repair-induction process. In the current study, DSB reduction by resveratrol was not very efficient; although resveratrol reduced the DSB level, it did not effectively lead to complete repair. In addition, the speed of the DSB reduction by resveratrol was slower than that of the general repair-induction process and the repair of DSBs caused by $\gamma$-rays $(2-5 \mathrm{~Gy})$, which usually takes a few hours $^{12,27}$, DSBs were typically not repaired $3 \mathrm{hr}$ after the resveratrol treatment. Reduction of the DSB level by resveratrol might involve chromatin remodelling. In fact, we identified a transient stabilisation of $\mathrm{H} 2 \mathrm{AX}$, which is mediated by the chromatin remodelling factors SNF2H and SIRT6 ${ }^{12}$, in resveratrol-treated MEFs. H2AX stabilization occurred between 1.5 and $6 \mathrm{hr}$ after resveratrol treatment and was reduced by $24 \mathrm{hr}$ post-treatment, corresponding to the time when the number of $\gamma \mathrm{H} 2 \mathrm{AX}$ foci was reduced.

In human, the peak serum/plasma concentrations of total resveratrol and its derivatives after ingestion of a single serving of red wine are typically lower than $1.5 \mu \mathrm{M}^{25}$. Although multiple effects of resveratrol have been reported in vitro, many, including its pro-apoptotic effect on cancer cells, were seen at concentrations much higher than those typically seen in serum ${ }^{43,44}$. The concentrations of resveratrol used in the current study (1.25$2.5 \mu \mathrm{M}$ ) might be unphysiologically high, but are much closer to those seen in serum/plasma than the concentrations used in previous studies. 
In summary, the results presented here suggest that regular consumption of resveratrol-associated polyphenols suppresses the incidence of cancers that arise with MSI by supporting DSB reduction and promoting genome stability.

\section{Methods}

Cell culture. MEFs were prepared from wild-type mice as described previously and were cultured using a Std-3T3 passage protocol ${ }^{45}$ or a modified protocol (Resv-3T3, Chlo-3T3 or MelRes-3T3), as described in the main text. To obtain immortalised cells, MEFs that reached the growth-arrested state (P8) were maintained without passaging, with a medium change every 3 days, until they exhibited immortal growth (IP1). All cells were cultured in Dulbecco's Modified Eagle's Medium (Nakarai) supplemented with $10 \%$ (v/v) foetal calf serum (Gibco).

Cell treatments and analyses. DSBs that arose spontaneously during growth of cells under the Std-3T3 protocol were monitored. Resveratrol (Sigma), chlorogenic acid (Sigma), gnetin C (Yamada Bee Company Inc.), gnemonoside A (Yamada Bee Company Inc.) and gnemonoside D (Yamada Bee Company Inc.) were used for cell treatments as indicated. Melinjo resveratrol (Yamada Bee Company Inc.), a powdered extract of melinjo seed (Lot.YMP-M-160121) standardised to contain a minimum of $20 \%$ resveratrol derivatives, was also used; the extract included $0.1 \%$ trans-resveratrol, $2.5 \%$ gnetin C, $19.6 \%$ gnemonoside A, $4.3 \%$ gnemonoside D and $9.0 \%$ dextrin. Western blotting was performed as described previously ${ }^{26}$ and proteins were transferred to PVDF membranes for $2 \mathrm{hr}$. Immunofluorescence analyses were performed as described previously ${ }^{26,27}$ using a confocal laser microscope (Olympus FV10i). $P$-values were used to determine the statistical significance of differences between groups. The $P$-values shown in dot plots and bar graphs were calculated via two-tailed Welch's $t$-tests.

Antibodies. Antibodies against the following proteins were obtained from the indicated suppliers: $\beta$-actin (AC-74, Sigma), H2AX (A300-082A-1 for immunofluorescence and A300-082A-2 for immunoblotting, Bethyl Laboratories), H3 (MABI0301, MBL), $\gamma \mathrm{H} 2 \mathrm{AX}$ (JBW301, Upstate Biotechnology; 9718, Cell Signaling Technology), PCNA (ab29, Abcam), p-RPA32 (phosphorylated at Ser 33) (NB100-544, Noxus Biologicals), 53BP1 (PC712, Merck), $\alpha$-tubulin (T6074, Sigma-Aldrich) and cleaved caspase-3 (9661, Cell Signaling Technology).

Animal experiments. Msh2 $2^{-1-}$ mice were fed the NMF non-purified diet (Oriental Yeast Co.) with or without $0.03 \%$ or $0.3 \%$ melinjo resveratrol (Yamada Bee Company Inc.). The mice were housed in plastic cages at a controlled temperature $\left(23^{\circ} \mathrm{C} \pm 3^{\circ} \mathrm{C}\right)$ and humidity $(50 \% \pm 10 \%)$ with a $12 \mathrm{hr}: 12 \mathrm{hr}$ light:dark cycle. In the intestinal polyp formation experiment, the mice were given drinking water containing $0.2 \% \mathrm{~K}_{2} \mathrm{BrO}_{3}$. All animal experiments were approved by the Animal Research and Animal Care Committee of Tohoku University (2014AgA-059, 2016AgA-022-1). The experiments were conducted under the guidelines issued by this committee, in accordance with Japanese governmental legislation (2005) that establishes rules for the care and use of animals in animal studies. $P$-values were used to determine the statistical significance of differences between groups. The Kaplan-Meir curve data were analysed by a Log-Rank test followed by a Holm-Sidak test. The tumour number was analysed via a Student's $t$-test.

Received: 3 September 2019; Accepted: 4 March 2020;

Published online: 25 March 2020

\section{References}

1. Lengauer, C., Kinzler, K. \& Vogelstein, B. Genetic instability in colorectal cancers. Nature 386, 623-627 (1997).

2. Matsuno, Y. et al. Replication stress triggers microsatellite destabilization and hypermutation leading to clonal expansion in vitro. Nat. Commun. 10, 3925 (2019).

3. Negrini, S., Gorgoulis, V. G. \& Halazonetis, T. D. Genomic instability - an evolving hallmark of cancer. Nature Reviews Molecular Cell Biology 11, 220-228 (2010).

4. Gorgoulis, V. G. et al. Activation of the DNA damage checkpoint and genomic instability in human precancerous lesions. Nature 434, 907-913 (2005).

5. Bartkova, J. et al. DNA damage response as a candidate anti-cancer barrier in early human tumorigenesis. Nature 434, $864-870$ (2005).

6. Bartkova, J. et al. Oncogene-induced senescence is part of the tumorigenesis barrier imposed by DNA damage checkpoints. Nature 444, 633-637 (2006).

7. Halazonetis, T. D., Gorgoulis, V. G. \& Bartek, J. An Oncogene-Induced DNA Damage Model for Cancer Development. Science 319, 1352-1355 (2008).

8. Ichijima, Y. et al. DNA lesions induced by replication stress trigger mitotic aberration and tetraploidy development. PLoS One 5, e8821 (2010).

9. Sedelnikova, O. A. et al. Senescing human cells and ageing mice accumulate DNA lesions with unrepairable double-strand breaks. Nat. Cell Biol. 6, 168-170 (2004).

10. Atsumi, Y. et al. Onset of Quiescence Following p53 Mediated Down-Regulation of H2AX in Normal Cells. PLoS One 6, e23432 (2011).

11. Osawa, T. et al. Arf and p53 act as guardians of a quiescent cellular state by protecting against immortalization of cells with stable genomes. Biochem. Biophys. Res. Commun. 432, 34-39 (2013).

12. Atsumi, Y. et al. ATM and SIRT6/SNF2H Mediate Transient H2AX Stabilization When DSBs Form by Blocking HUWE1 to Allow Efficient $\gamma$ H2AX Foci Formation. Cell Rep. 13, 2728-2740 (2015).

13. Howitz, K. T. et al. Small molecule activators of sirtuins extend Saccharomyces cerevisiae lifespan. Nature 425, 191-196 (2003).

14. Wood, J. G. et al. Sirtuin activators mimic caloric restriction and delay ageing in metazoans. Nature 430, 686-689 (2004).

15. Valenzano, D. R. et al. Resveratrol prolongs lifespan and retards the onset of age-related markers in a short-lived vertebrate. Curr. Biol. 16, 296-300 (2006).

16. Yu, X. \& Li, G. Effects of resveratrol on longevity, cognitive ability and aging-related histological markers in the annual fish Nothobranchius guentheri. Exp. Gerontol. 47, 940-949 (2012).

17. Rascón, B., Hubbard, B. P., Sinclair, D. A. \& Amdam, G. V. The lifespan extension effects of resveratrol are conserved in the honey bee and may be driven by a mechanism related to caloric restriction. Aging (Albany. NY). 4, 499-508 (2012). 
18. Bitterman, J. L. \& Chung, J. H. Metabolic effects of resveratrol: Addressing the controversies. Cell. Mol. Life Sci. 72, 1473-1488 (2015).

19. Soleas, G. J., Grass, L., Josephy, P. D., Goldberg, D. M. \& Diamandis, E. P. A comparison of the anticarcinogenic properties of four red wine polyphenols. Clin. Biochem. 35, 119-124 (2002).

20. Harper, C. E. et al. Resveratrol suppresses prostate cancer progression in transgenic mice. Carcinogenesis 28, 1946-1953 (2007).

21. Sengottuvelan, M. \& Nalini, N. Dietary supplementation of resveratrol suppresses colonic tumour incidence in 1,2-dimethylhydrazine-treated rats by modulating biotransforming enzymes and aberrant crypt foci development. Br. J. Nutr. 96, 145 (2006).

22. Banerjee, S., Bueso-Ramos, C. \& Aggarwal, B. B. Suppression of 7,12-dimethylbenz(a)anthracene-induced mammary carcinogenesis in rats by resveratrol: Role of nuclear factor- $\kappa B$, cyclooxygenase 2 , and matrix metalloprotease 9. Cancer Res. 62, 4945-4954 (2002).

23. Matheu, A. et al. Delayed ageing through damage protection by the Arf/p53 pathway. Nature 448, 375-379 (2007).

24. Minakawa, Y., Shimizu, A., Matsuno, Y. \& Yoshioka, K. Genomic Destabilization Triggered by Replication Stress during Senescence. Cancers 9, 159 (2017).

25. Baur, J. A. \& Sinclair, D. A. Therapeutic potential of resveratrol: The in vivo evidence. Nature Reviews Drug Discovery 5, 493-506 (2006).

26. Atsumi, Y. et al. The Arf/p53 Protein Module, Which Induces Apoptosis, Down-regulates Histone H2AX to Allow Normal Cells to Survive in the Presence of Anti-cancer Drugs. J. Biol. Chem. 288, 13269-13277 (2013).

27. Minakawa, Y., Atsumi, Y., Shinohara, A., Murakami, Y. \& Yoshioka, K. Gamma-irradiated quiescent cells repair directly induced double-strand breaks but accumulate persistent double-strand breaks during subsequent DNA replication. Genes to Cells 21, 789-797 (2016).

28. Zheng, S.-Q. et al. Chlorogenic Acid Extends the Lifespan of Caenorhabditis elegans via Insulin/IGF-1 Signaling Pathway. Journals Gerontol. Ser. A Biol. Sci. Med. Sci. 72, glw105 (2016).

29. Sur, S. \& Panda, C. K. Molecular aspects of cancer chemopreventive and therapeutic efficacies of tea and tea polyphenols. Nutrition 43-44, 8-15 (2017).

30. Tajik, N., Tajik, M., Mack, I. \& Enck, P. The potential effects of chlorogenic acid, the main phenolic components in coffee, on health: a comprehensive review of the literature. Eur. J. Nutr. 56, 2215-2244 (2017).

31. Tani, H. et al. Pharmacokinetics and Safety of Resveratrol Derivatives in Humans after Oral Administration of Melinjo (Gnetum gnemon L.) Seed Extract Powder. J. Agric. Food Chem. 62, 1999-2007 (2014).

32. Hombauer, H., Srivatsan, A., Putnam, C. D. \& Kolodner, R. D. Mismatch repair, but not heteroduplex rejection, is temporally coupled to DNA replication. Science 334, 1713-6 (2011).

33. Hombauer, H., Campbell, C. S., Smith, C. E., Desai, A. \& Kolodner, R. D. Visualization of Eukaryotic DNA Mismatch Repair Reveals Distinct Recognition and Repair Intermediates. Cell 147, 1040-1053 (2011).

34. De Wind, N., Dekker, M., Berns, A., Radman, M. \& Te Riele, H. Inactivation of the Mouse Msh2 Gene Results in Mismatch Repair Deficiency, Methylation Tolerance, Hyperrecombination, and Predisposition to Cancer. Cell 82 (1995).

35. Bishayee, A. \& Dhir, N. Resveratrol-mediated chemoprevention of diethylnitrosamine-initiated hepatocarcinogenesis: Inhibition of cell proliferation and induction of apoptosis. Chem. Biol. Interact. 179, 131-144 (2009).

36. Noh, K. T. et al. Resveratrol suppresses tumor progression via the regulation of indoleamine 2,3-dioxygenase. Biochem. Biophys. Res. Commun. 431, 348-353 (2013).

37. Piao, J., Nakatsu, Y., Ohno, M., Taguchi, K. \& Tsuzuki, T. Mismatch repair deficient mice show susceptibility to oxidative stressinduced intestinal carcinogenesis. Int. J. Biol. Sci. 10, 73-9 (2013).

38. Tomasetti, C. et al. Variation in cancer risk among tissues can be explained by the number of stem cell divisions. Science 347, 78-81 (2015).

39. Wu, S., Powers, S., Zhu, W. \& Hannun, Y. A. Substantial contribution of extrinsic risk factors to cancer development. Nature 529, 43-47 (2016).

40. Tomasetti, C. et al. Role of stem-cell divisions in cancer risk. Nature 548, E13-E14 (2017).

41. Tomasetti, C., Li, L. \& Vogelstein, B. Stem cell divisions, somatic mutations, cancer etiology, and cancer prevention. Science 355, $1330-1334$ (2017).

42. Yoshioka, K., Matsuno, Y., Hyodo, M. \& Fujimori, H. Genomic-Destabilization-Associated Mutagenesis and Clonal Evolution of Cells with Mutations in Tumor-Suppressor Genes. Cancers 11, 1643 (2019).

43. Tang, Q. et al. Resveratrol-induced apoptosis is enhanced by inhibition of autophagy in esophageal squamous cell carcinoma. Cancer Lett. 336, 325-337 (2013).

44. Chang, C.-H. et al. Resveratrol-induced autophagy and apoptosis in cisplatin-resistant human oral cancer CAR cells: A key role of AMPK and Akt/mTOR signaling. Int. J. Oncol. 50, 873-882 (2017).

45. Todaro, G., Green, H., Torado, G. J. \& Green, M. D. Quantitative Studies of The Growth of Mouse Embryo Cells in Culture and Their Development Into Established Lines. J. Cell Biol. 17, 299-313 (1963).

\section{Acknowledgements}

This study is supported by Yamada Research Grant, Japan Coffee Association, The Tojuro Iijima Foundation for Food Science and Technology, Mishima Kaiun Memorial Foundation, and the JSPS Core-to-Core Program A (Advanced Research Networks) entitled: "Establishment of international agricultural immunology research-core for a quantum improvement in food safety". This work is also supported by Yamada Bee Company Inc.

\section{Author contributions}

Y.M., Y.A., H.F., M.H., and A.S., designed the in vitro work, performed experiments, and analysed the data; M.A. and M,M.R. designed the in vivo work and performed experiments, interpreted the data; T.I. and H.Tani. prepared samples and analysed the data; H.Torigoe, Y.N., T.T., and M.K. interpreted the data; H.S. designed the in vivo work and interpreted the data and wrote the manuscript; K.Y. designed and supervised the project, performed experiments, interpreted the data, and wrote the manuscript.

\section{Competing interests}

T.I. and H.Tani are employees of Yamada Bee Company Inc. All other authors have no competing interests.

\section{Additional information}

Supplementary information is available for this paper at https://doi.org/10.1038/s41598-020-62292-5.

Correspondence and requests for materials should be addressed to K.-i.Y. 
Reprints and permissions information is available at www.nature.com/reprints.

Publisher's note Springer Nature remains neutral with regard to jurisdictional claims in published maps and institutional affiliations.

(c) (i) Open Access This article is licensed under a Creative Commons Attribution 4.0 International License, which permits use, sharing, adaptation, distribution and reproduction in any medium or format, as long as you give appropriate credit to the original author(s) and the source, provide a link to the Creative Commons license, and indicate if changes were made. The images or other third party material in this article are included in the article's Creative Commons license, unless indicated otherwise in a credit line to the material. If material is not included in the article's Creative Commons license and your intended use is not permitted by statutory regulation or exceeds the permitted use, you will need to obtain permission directly from the copyright holder. To view a copy of this license, visit http://creativecommons.org/licenses/by/4.0/.

(C) The Author(s) 2020 